\title{
Autonomous sailboats: an emerging technology for ocean sampling and surveillance
}

\author{
Nuno A. Cruz and José Carlos Alves \\ Faculdade de Engenharia da Universidade do Porto \\ Rua Dr. Roberto Frias, 4200-465, Porto, Portugal \\ Email: $\{$ nacruz, jca $\} @$ fe.up.pt
}

\begin{abstract}
Autonomous sailboats are robotic vessels that use wind energy for propulsion and control the sails and rudders without human intervention. The use of autonomous sailboats for ocean sampling has been tentatively proposed before, but there have been minor efforts towards the development and deployment of actual prototypes, due to a number of technical limitations and significant risks of operation. Currently, most of the limitations have been surpassed, with the availability of extremely low power electronics, flexible computational systems, reliable communication devices and high performance renewable power sources. At the same time, some of the major risks have been mitigated, allowing this emerging technology to become an effective tool for a wide range of applications in real scenarios. We illustrate some of these scenarios and we describe the status of the current efforts being made to develop operational prototypes.
\end{abstract}

\section{INTRODUCTION}

Sailing is a relatively complex task, dependant on unpredictable environmental conditions such as wind and sea state. In conventional sailing boats, the sailor controls the rudder according to the desired course and uses the sail sheet to maximize velocity. For a given course, boat speed, wind speed and wind direction, there is an optimum angle between the sail and direction of the wind that maximizes the speed of the boat.

Autonomous sailboats are robotic vessels that use wind energy for propulsion and have the capability to control the sails and rudders without human intervention. There has been some significant work in the automation of sailing tasks, particularly in what concerns navigation for single handed sailors [1][2]. However, the natural evolution to the development of fully autonomous sailboats for ocean sampling has suffered from a number of technical limitations and significant risks of operation. In this paper, we show that currently, most of the limitations have been surpassed, with the existing availability of extremely low power electronics, flexible computational systems and high performance renewable power sources. At the same time, some of the major risks have been mitigated, allowing this emerging technology to become an effective tool for a wide range of applications in real scenarios, complementing the other technologies available for ocean sampling.

This paper is organized as follows. Section II provides some background regarding ocean sampling, briefly introducing available technology and methods to obtain ocean data. In Section III we provide a typical SWOT analysis (Strengths, Weaknesses, Opportunities and Threats) relative to the potential utilization of autonomous sailboats in the ocean.
Next, in Section IV, we describe a set of applications where autonomous sailboats may be effectively used. In Section V we analyze the status of the current efforts being made to develop fully operational prototypes, and finally, Section VI presents the main conclusions and the plans for the near future.

\section{Motivation}

\section{A. The importance of (more) ocean data}

In the last decades, the oceanographic community has started relying on ocean robotics to collect in-situ data and, recently, there has been an exponential increase in the utilization of state of the art technology, such as autonomous underwater vehicles, underwater gliders/profilers and unmanned surface vehicles. At the same time, there has been a dramatic broadening on the knowledge about the physical processes behind the ocean dynamics. This has allowed for the development of various mathematical models that attempt to reproduce the real conditions found in the oceans. Although these models require calibration with real multi-scale data, the fact is that the data available has always been relatively scarce, particularly what concerns in-situ measurements [3].

Similarly, coastal management authorities can also benefit from any technological advances in ocean surveillance, in order to complement the solutions already in use to fight illegal activities, particularly trading and emigration.

\section{B. Available technology and methods - a brief survey}

Moored Instrumentation and Ocean Observatories - Insitu long term observatories are important tools for monitoring time-variations of oceanic processes. Ocean observatories are unmanned systems located at a fixed site, providing information regarding the seafloor, the water column and the surface. They have been installed all over the world, particularly in the last decade [4]. Arrays of moored, and therefore static, instrumentation can provide simultaneous time series, but spatial resolution is typically poor due to the high cost. Current developments try to get the most out of the moored instrumentation by combining a profiling mechanism to a moored based system [5].

Ships of opportunity - One of the easiest ways to get large-scale ocean measurements is the installation of in-situ instruments on ships of opportunity, such as cargo ships or ferry-boats [6]. Typical sensors include temperature and conductivity recorders, current profilers, etc. In these systems, 
data is stored internally and it is only retrieved when the ship reaches the final destination or completes a round trip.

Towed Systems - Towed bodies can provide quasi-synoptic two-dimensional sections of the evolving ocean fields. They are controlled from a ship via an umbilical cord, providing power and communications. They have limited maneuverability and can follow simple trajectories, using deflection surfaces for changing depth and orientation. Some undulating versions allow for complex vertical patterns, such as yo-yo's.

Remotely Operated Vehicles (ROVs) - ROVs are underwater robotic machines that operate with a physical link with the surface. Nonetheless all technological advances, the mobility of these vehicles remain severely constrained by the umbilical, and the drag associated to the frame and the cable prevent high velocities, so that the major application for these vehicles is to perform close inspections in environments with low currents, in shallow waters.

Drifters, Profilers and Gliders - Drifters are instrumented floats dropped from a support vessel, which then drift horizontally with the local currents for long periods of time [4]. Present profilers are similar to the drifters, but they use variable buoyancy to move vertically through the ocean. Gliders not only use variable buoyancy to move vertically, but they have also wings and control foils designed to allow steerable gliding, thus providing for some limited control on horizontal propulsion [7]. Some of these vehicles can surface from time to time, get a GPS position and communicate through appropriate satellite links. Nonetheless, the amount of information that can be transmitted is very limited and the scientists need to wait for recovery to get full data. For a long time, there have been suggestions for the harvest of propulsion energy from the environment to allow for very long range operations, and recently a heat engine that draws energy from the ocean thermocline has been tested [8].

Autonomous Surface Vehicles (ASVs) - ASVs are robotic boats that operate without any physical link with the operator. ASVs have usually a large capacity in terms of payload, but the limited amount of energy stored on board prevents their use in long range missions. Although there has been some effort regarding the deployment of ASVs for operation in coastal waters, the fact is that most of the prototypes being developed are mainly intended for calm inland waters [9][10].

Autonomous Underwater Vehicles (AUVs) - In the last decade, some important technological advances, together with new ideas for efficient ocean sampling, fostered the development of AUVs [11]. AUVs constitute powerful and effective tools for underwater data gathering and there is a large number of operational systems, in various sizes and configurations, covering commercial, military or scientific applications [11] [12][13]. These vehicles operate with no physical link with the surface, carrying a set of relevant sensors to characterize the underwater environment. AUV utilization is still quite limited as far as routine ocean sampling is concerned, but some very interesting results have aroused from their use in challenging environments, such as under Arctic ice-sheets, in deep water, in very shallow waters, and in extreme environments.
Remote Sensing - Due to their global coverage and sophisticated instrumentation, environmental satellites are playing an expanding role in monitoring ocean conditions, namely in sea-surface temperature and chlorophyll concentration. The contributions of satellites are fundamental to measuring variations on time scales ranging from seasonal-interannual to decadal. However, they lack in detail and have poor vertical information.

\section{The AOSN Concept}

The concept of Autonomous Ocean Sampling Networks (AOSN) was introduced in [14], in a novel approach to provide a framework to encompass a set of cooperative efforts taking place, integrating multiple information sources about the oceans.

The fundamental idea behind this paradigm is the cooperative utilization of the complementary technology available for ocean sampling, in order to provide a synergetic observation system for a given region. Curtin et al. envisaged the installation of moored instrumentation linked to shore via radio or satellite communications, providing oceanographic and atmospheric data in real time. At the same time, a set of small, high-performance vehicles (gliders and AUVs) would be navigating in the area to provide intensive 4-D data about the region.

\section{Why autonomous sailboats?}

One of the characteristics of autonomous robotic systems is the absence of physical connections with any operator, therefore they have to carry all required energy or/and harvest some energy from the environment. For any moving system, the fraction of energy necessary to provide motion is usually significant. Autonomous sailboats rely on wind to provide propulsion and so they only need electrical energy for the onboard electronics and rudder adjustments. With current reduction in power consumption from electronic circuits and sensors, it is possible to trim down the energy requirement to a few tens of Watt-hour per day. At the same time, there have been major developments in technology associated with renewable energy sources for micro-generation, such as miniature wind turbines and solar panels, and it is now possible to have high performance commercial-off-the-shelf energy generators at very reasonable costs [15]. If we combine this with the energy densities provided by new battery technologies, such as Lithium-Ion, then it is clear that it is feasible to devise an energy management system that can provide a continuous supply of power to the onboard electronics.

Autonomous sailboats can transport a wide variety of sensors and store the incoming data internally or transmit it to shore via radio or satellite. Even the smallest autonomous sailboats have some space available for sensors, either in the hull, in the mast or in the form of an underwater towed system. With the ability to travel for long distances, even though it may be at modest velocities, these systems may provide valuable ocean data in spacial and temporal scales complementary to the other technologies already in use. 


\section{E. Dimensions of autonomous sailboats}

The size of a recreational yacht varies from the most modest single person "dinghy" to the long, luxury models. Besides personal preferences (wether aesthetics, social impact, or other), size is mainly dictated by a tradeoff between comfort and price. When it comes to autonomous sailboats, there are no limitations regarding people transportation and surely comfort is not an issue. Many other aspects have to be contemplated instead, and usually the driving factors are safety and performance. Sailing performance results from a complex tradeoff between various design factors, such as sail area, sail shape, hull size and hull shape [16].

When determining the size of an autonomous sailboat, it is also important to consider both the permanent hardware that needs to be installed (electronics and mechanical systems) and also the extra payload that may be transported for particular applications. Even with these constraints, there is usually some degree of flexibility on the overall size of the sailboat, providing the scaling process is taken according to the principles of yacht design [16], [17].

The main benefits of building a larger sailboat are:

- More velocity - Theoretically, the maximum velocity of a sailboat is proportional to the square-root of the length on the water line (LWL);

- More payload capacity - The available volume inside the hull increases with the cube of the scaling factor. A bigger sailboat will also have a higher mast and a larger deck space for sensors and electronics;

- More stability - As the dimensions increase, it is possible to improve the ratio between the ballast and the total weight, increasing stability.

A larger sailboat has also some inconveniences, such as:

- Cost increase - The cost of hull construction naturally increases for a larger scale;

- More complex logistics - An increase in size and weight impair storage, transportation and operational logistics;

- More power required for steering - A larger, heavier sailboat demands more power for steering.

Another consequence of increasing the size of a sailboat is to augment the visibility as seen from other ships. This may be an advantage when the priority goes to equipment safety (diminishing the risks of ship collision), and/or when a surveillance operation also relies on the deterrence ability. In other surveillance scenarios, however, it may be preferable to have a virtually invisible sailboat, and in this case a smaller size is preferable.

\section{The Role of Autonomous Sailboats - A SWOT APPROACH}

\section{A. Strengths}

Long mission ranges - Assuming that every autonomous sailboat has an energy management system capable of charging a set of internal batteries, then these vehicles have practically no limitations in range and so they can be used for long term, large scale in-situ data sampling or permanent surveillance.
Negligible operational costs - The costs of operating an autonomous sailboat are essentially those associated with the support infrastructure, such as communications, backing personnel and hypothetical emergency rescue equipment.

Potential for towing sensors - Autonomous sailboats have no underwater moving parts, apart from the small rudders in the stern, which have a slow and only occasional activity. Thus, it is extremely easy to tow external sensors and/or arrays without the risk of the sensor cables getting tangled. With minor effort, it is even possible to conceive a winchdriven system that can be lowered in the water column in calm regions.

Real time data transmission - Autonomous sailboats can use a radio or satellite link to transmit sensor data to a control station. This information may be interpreted by a mission coordinator to periodically assess the quality of the sensor data, to alter the trajectory or to react in some other way.

Real time localization - Using standard (and inexpensive) commercial off-the-shelf technology, it is possible for an onboard computer to know the exact location anywhere in the world, and relay this information back to a control station via radio. It is also relatively easy to use redundant tracking devices, such as satellite-based, to know the position of the sailboat.

Very low noise generation - Autonomous sailboats generate a minor amount of acoustic noise as compared to motorized vessels, with virtually no noise produced by propulsion and only a small amount originated in the interaction between the hull and the water. With a proper mechanical design, the other sources of underwater noise may be neglected, such as sail and cable vibration, small rudder corrections, etc. Thus, with respect to sound detection, these sailboats are comparable in performance to gliders and drifters.

\section{B. Weaknesses}

Risk of collision - The smallest sailboats may be hard to detect from a large ship radar, so there is a serious risk of ship collision, particularly when crossing regions with large ship traffic. There is also a great number of floating debris in the ocean and it is possible that an hypothetical collision with a large fragment cause serious damage to the hull of an autonomous sailboat, particularly for small size vehicles.

Vulnerability to extreme weather conditions - When a vessel is programmed to travel hundreds or even thousands of miles, it is very likely to find high seas and bad weather at any moment during the journey. With the current capabilities of weather forecasting, it is usually possible to predict an incoming storm several days in advance, which may be useful to change the course of the sailboat using satellite communications. Nonetheless, if the wind does not help, it may be impossible to run away from an incoming storm.

Limited access to the ocean - Autonomous sailboats travel at the surface of the ocean, and so they are well suited to measure surface or sub-surface data. Even with towed or winch-driven systems, it is not expected that these vehicles can sample more than the very top layer of the ocean. 
Degradation of sensor accuracy over time - Bio-fouling is a nuisance associated with any system subject to long term deployments, and it is particularly severe in the ocean. In the case of oceanographic optic sensors, there are already a few products with a very small wiper that periodically cleans the sensing window. However, the great majority of oceanographic sensors require regular maintenance to remove any growth and recalibration to ensure the specified accuracy. Overall, one should not expect the same accuracy from long term sensor installations as compared to data from manual in-situ measurements or laboratory analysis, for example.

Exposure to vandalism - Autonomous sailboats are usually unguarded and so they may be easy targets for vandalism, particularly close to shore. This risk may be reduced with cameras installed on board and warning signs.

Impossibility of fixing a velocity - One inconvenience of using autonomous sailboats for ocean surveys is that it is impossible to stipulate a-priori the velocity, since it depends on the wind and sea state conditions. Therefore, it is not possible to predict the time that the boat will take to complete any given trajectory, usually given as a set of waypoints.

\section{Opportunities}

Real scenarios for current prototypes - Given the possibility of transporting oceanographic sensors during very long missions, autonomous sailboats can play an important role in ocean-scale sampling. The opportunity to work 24 hours a day and transport radars and cameras (visual and infrared) make these vehicles a possible tool for coastal surveillance. Some of these applications will be detailed in the next section.

Future applications - The prototypes that are currently being developed are small scale models, not only in physical size, but also in the number of automated features. They are mainly intended to demonstrate the feasibility of autonomous sailing, with little interest in mimicking the actions performed by a sailor in a real yacht. With the development of full scale models, it will be possible for a computer to control a greater number of sailing actions (fold/unfold multiple sails, compensate the onboard weight distribution, etc.), so that the prototypes may be used to test different sailing strategies in the field. Ultimately, the ability to replace a human sailor in a standard sailboat may also be useful for handicapped people, extending what is currently defined as autopilot.

\section{Threats}

Absence of applicable legislation - There is currently an absence of international legislation regarding the navigation of autonomous systems in the ocean [18]. An hypothetical restraining international legislation may completely forbid the deployment of such vessels, making all technological efforts useless, or it may also happen that different countries decide differently regarding this subject. Recently, in an attempt to anticipate these legal issues, there have been some efforts towards the implementation of behaviors that comply with standard marine regulations into autonomous marine vessels, particularly in what concerns collision avoidance [19][20].
Demonstration failure - Although all required subsystems have been separately demonstrated, a fully autonomous sailboat has yet to be validated in a real scenario. In a first stage, it is crucial to have a sailboat consistently navigating through the predefined marks and harvesting energy from the wind and/or the sun for a significant length of time. Then, it is important to repeat the test under severe conditions to assess the robustness of the mechanical structure (hull, mast, keel, cables, etc.) and electronics in an extremely harsh environment.

\section{Potencial apPlications of AUtonomous SAILBOATS}

\section{A. Ocean Observation}

Upper Ocean Dynamics - The dynamics of both the ocean and the atmosphere are mainly determined by the energy they exchange. Oceanographers have been studying the processes that occur in the top layer of the ocean (eddies, fronts, meanders, etc), since they are extremely important to define how this exchange occurs and, at the same time, are affected by the climatology of the atmosphere. Instrumented sailboats may be an important tool to contribute to the understanding of this interaction, as they can gather relevant data (both hydrological and atmospheric parameters), precisely at this boundary layer.

Ocean circulation - The study of the ocean circulation has direct impact in many different processes, such as biological activity and climate variability [21]. Typically, circulation studies encompass multi-scale measurements and therefore these investigations can be supported by long-range autonomous sailboats. For this application, the sailboats should be equipped with acoustic doppler current profilers, with the capability to measure the oceans' currents from the surface down to 1000 meters of depth. Although these devices require significant power for each measurement, they can be programmed to work at very low duty-cycles.

Chlorophyll concentration - The chlorophyll concentration is important to estimate the amount and distribution of phytoplankton in the ocean, which is the basis of the ocean food chain. Phytoplankton grow by photosynthesis, a process which consumes carbon dioxide, and so they are also important in the ocean carbon cycle and, consequently, influence the greenhouse effect and climate change. Chlorophyll concentration is regularly obtained for the ocean surface by satellite measurements [22]. However, the scale is very coarse and it is important to complement the satellite observations and provide some means of periodically calibrate the satellite data. This can be made by in-situ measurements of chlorophyll, using fluorometers installed in autonomous sailboats.

Ocean acoustics - The fact that autonomous sailboats generate almost no self noise makes them suitable for acoustic measurements in the ocean. These vehicles may transport hydrophones with a wide bandwidth (either omnidirectional or directional) and record acoustic activity throughout the journey. Currently, these measurements are routinely carried out to detect mammal sounds (such as whales, for example) using drifters, gliders or AUVs [23]. 
Tracking pollution plumes - Satellite images are already being used to follow the evolution of pollution plumes in the ocean, which is particularly relevant in coastal areas [24]. However, this information has very low resolution and only gives data in 2 dimensions. Autonomous sailboats can transport hydrocarbon sensors together with towed sensors (dissolved oxygen or chlorophyll, for example) to monitor the upper layer of the ocean, measuring the thickness and the concentration of the pollution layer. They can also measure atmospheric conditions (local winds, air temperature, etc.) that may influence the evolution of the plumes.

Calibration of basin-wide ocean models - Recent advances in the understanding of the processes governing the dynamics of the oceans have fostered the development of ocean forecast models [25]. Autonomous sailboats can provide these models with data from real in-situ observations, taken at multiple temporal and spatial scales.

\section{B. Coastal surveillance}

Detection and prevention of illegal trading - Illegal trading routes often include maritime itineraries, and so coastal surveillance is essential to mitigate this problem. If we consider an average velocity of 3 knots, then a sailboat can travel about 70 miles per day. Surely that illegal traders use extremely fast speed boats, but a clever distribution of sailboats along the coast, together with the installation of $360^{\circ}$ cameras, may prove to be an effective tool for detection of illegal activities and trigger further actions from the relevant authorities. With sufficient media hype, emphasizing the random nature of sailboat location, these vehicles can also act as effective deterrence tools against illegal trading.

Surveillance of immigration routes - There have been several recent episodes of casualties in ill-equipped crafts overloaded with illegal immigrants. Autonomous sailboats distributed along the coast with visible and infrared cameras may guarantee a permanent presence, 24 hours a day. This is a scenario for which it is important to design sailboats that can withstand bad weather and high seas, since these conditions are the most critical when it comes to people safety.

\section{Military applications}

Mine countermeasures - Autonomous sailboats may be used close to shore for mine detection using sonars like sidescan, multibeam, or synthetic aperture. This prevents operators to approach a potential minefield, and may also provide high resolution hydrographic data. This type of mission is already being conducted with AUVs [12], but the advantage of using autonomous sailboats is that the data can be transmitted in real time to a mother ship, along with an accurate absolute positioning given by (D)GPS. With a permanent team continuously analyzing the data, it is possible to validate the targets and go back to the same location in case of doubt.

Enemy coastal survey - One or more autonomous sailboats may be launched from a mother ship and approach shore with visual or infrared cameras mounted on top of the mast. Since autonomous sailboats have reduced power dissipation on board, it is expected that their own infrared signature be minimal and therefore they should be able to conduct surveys virtually unnoticed.

\section{Status of CurRent EFForts}

\section{A. Autonomous Sailboats Initiatives}

Probably the most important initiative to promote the development of autonomous sailboats is the Microtransat challenge, an annual competition that has as an ultimate goal to cross the Atlantic Ocean. The Microtransat was first organized in Toulouse, France, in June 2006, and the $3^{\text {rd }}$ competition, in May 2008, included the first World Robotic Sailing Competition, in the lake Neusiedl, Austria. This competition had the participation of four teams (Austria, Canada, Portugal and Wales), and the sailboats showed the ability to navigate autonomously through a sequence of waypoints and complete the regattas. The Atlantic crossing is planned for the Autumn of 2008, leaving from the Portuguese coast, with 10 teams already registered to compete.

Across the Atlantic, a similar initiative has been hosted in Canada, with Sailbot being organized in June 2006 by Queen's University. The second edition, initially scheduled for 2007 , was later canceled and transferred to 2008.

Overall, there are very few scientific publications regarding the development or deployment of autonomous robotic sailboats, since most of the current projects are at an early stage and some other older projects were discontinued. Anyway, there is a great number of enthusiastic people involved in the current projects (with a significant number of students), and some teams are already being supported by sponsoring companies, which is a good demonstration of the current interest in this new technology.

\section{B. The FASt project}

The FEUP ${ }^{1}$ autonomous sailboat (FASt) is a small scale sailing yacht capable of fully autonomous navigation through a predefined set of marks (Fig. 1).

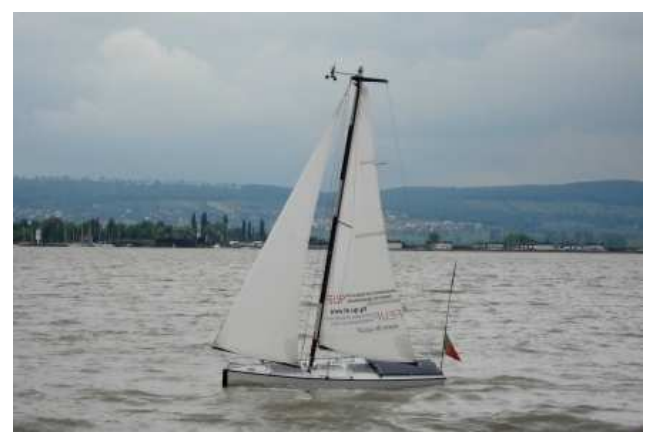

Fig. 1. FASt - the FEUP Autonomous Sailboat.

The FASt project was launched in the beginning of 2007 with two major objectives in mind: minimize the energy required for sailing and provide an efficient sailing boat

\footnotetext{
${ }^{1}$ Faculty of Engineering at the University of Porto
} 
capable of autonomous navigation under a broad range of weather conditions. This boat was custom designed and built by a team of professors and students at FEUP, and it is a flexible autonomous navigation platform, being able to carry a few kilograms of payload equipment.

The design length was set to 2.5 meters, after scaling down, in length and displacement, some modern designs of oceanic sailing boats and keeping the total weight relatively low to facilitate the launch and transportation, either by towing or on the top of a car. To increase stability, the boat has a deep keel with a lead ballast. The rig is a conventional Marconi configuration with a headsail rigged on a small boom, as used in smaller RC sailing boats. Main dimensions are presented in table I.

TABLE I

MAIN DIMENSIONS OF THE FEUP AUtONOMOUS SAILBOAT - FAST

\begin{tabular}{|l|c|}
\hline Total length (LOA) & $2.50 \mathrm{~m}$ \\
\hline Length in the water line (LWL) & $2.48 \mathrm{~m}$ \\
\hline Maximum width (beam) & $0.67 \mathrm{~m}$ \\
\hline Draft & $1.25 \mathrm{~m}$ \\
\hline Displacement & $50 \mathrm{Kg}$ \\
\hline Wet surface & $1.0 \mathrm{~m}^{2}$ \\
\hline Ballast & $20 \mathrm{Kg}$ \\
\hline Sail area & $3.7 \mathrm{~m}^{2}$ \\
\hline Mast height & $3.4 \mathrm{~m}$ \\
\hline
\end{tabular}

\section{CONCLUSIONS AND FUTURE WORK}

Until very recently, most of the efforts in developing autonomous sailboats were conducted towards the engineering challenges, either in electronics, mechanics or software programming. Now, the first prototypes are showing that most of these challenges were overcome and it is already possible to build fully autonomous sailboats using high-performance computers and low power electronics. At the same time, a combination of high density batteries with efficient renewable power sources allows for the installation of an energy management systems with indefinite duration. With such a system in mind, we have identified a set of applications for which the utilization of autonomous sailboats may prove to be both effective and efficient.

Autonomous sailboats are already being tested successfully in real scenarios, and so in the near future it will be possible to demonstrate their effectiveness for ocean sampling or surveillance in some of the applications described.

There is a regulatory gap in current international maritime law in what concerns the deployment of these vessels, since the international rules for collision avoidance only address boats under human control. The deployment of these autonomous sailboats will have to comply to some sort of standardized regulations, for which the availability of new electronic devices, such as AIS, may be a valuable asset.

\section{ACKNOWLEDGEMENTS}

The authors would like to acknowledge the support from the Department of Electrical and Computer Engineering at FEUP and the companies sponsoring the construction of FASt.

\section{REFERENCES}

[1] W. H. Warden, "A control system model for autonomous sailboat navigation," in IEEE Proc. of Southeastcon'91, Williamsburg, VA, USA, Apr. 1991, pp. 944-947.

[2] M. L. van Aartrijk, C. P. Tagliola, and P. W. Adriaans, "AI on the ocean: the RoboSail project," in Proc. 15th Eur. Conf. On Art. Int. ECAI'2002, Lyon, France, Jul. 2002, pp. 653-657.

[3] J. Legrand, M. Alfonso, R. Bozzano, G. Goasguen, H. Lindh, A. Ribotti, I. Rodrigues, and C. Tziavos, "Monitoring the marine environment operational practices in europe," in Proc. Int. Conf. EuroGOOS, 2003.

[4] N. N. Soreide, C. E. Woody, and S. M. Holt, "Overview of ocean based buoys and drifters: Present applications and future needs," in Proc. MTS/IEEE Conf. Oceans'01, Honolulu, HI, USA, Nov. 2001.

[5] M. Brown, M. Kelley, and P. McGill, "MBARI vertical profiler," in Proc. MTS/IEEE Conf. Oceans'01, Honolulu, HI, USA, Nov. 2001.

[6] W. Petersen, M. Petschatnikov, F. Schroeder, and H. Wehde, "Application of a ferrybox: Automatic measurements in the north sea," in Proc. MTS/IEEE Techno-Oceans'04, Kobe, Japan, 2004.

[7] C. C. Eriksen, T. J. Osse, R. D. Light, T. Wen, T. W. Lehman, P. L. Sabin, J. W. Ballard, and A. M. Chiodi, "Seaglider: A long-range autonomous underwater vehicle for oceanographic research," IEEE $J$. Oceanic Engineering, vol. 26, no. 4, pp. 424-436, Oct. 2001.

[8] D. C. Webb, P. J. Simonetti, and C. P. Jones, "SLOCUM: An underwater glider propelled by environmental energy," IEEE J. Oceanic Engineering, vol. 26, no. 4, pp. 447-452, Oct. 2001.

[9] J. Curcio, J. Leonard, and A. Patrikalakis, "SCOUT - a low cost autonomous surface platform for research in cooperative autonomy," in Proc. MTS/IEEE Conf. Oceans'05, Washington, D.C., USA, Sep. 2005.

[10] N. Cruz, A. Matos, S. Cunha, and S. Silva, "Zarco - an autonomous craft for underwater surveys," in Proc. 7th Geomatic Week, Barcelona, Spain, Feb. 2007.

[11] G. Griffiths, Ed., Technology and Applications of Autonomous Underwater Vehicles. UK: Taylor and Francis, 2003.

[12] C. von Alt, B. Allen, T. Austin, N. Forrester, R. Goldsborough, M. Purcell, and R. Stokey, "Hunting for mines with REMUS: A high performance, affordable, free swimming underwater robot," in Proc. MTS/IEEE Conf. Oceans'01, Honolulu, HI, USA, Nov. 2001.

[13] N. A. Cruz and A. C. Matos, "The MARES AUV, a modular autonomous robot for environment sampling," in Proc. MTS/IEEE Conf. Oceans'08, Quebec, Canada, Sep. 2008.

[14] T. Curtin, J. Bellingham, J. Catapovic, and D. Webb, "Autonomous oceanographic sampling networks," Oceanography, vol. 6, no. 3, pp. 86-94, 1993.

[15] P. Maycock and T. Bradford, "PV market update: Demand grows quickly and supply races to catch up," Renewable Energy World, vol. 10, no. 4, 2007.

[16] C. A. Marchaj, Sail Performance: Techniques to Maximize Sail Power. International Marine Publishing, 1996.

[17] N. L. Skene, Elements of Yacht Design. USA: Sheridan House, 2001.

[18] S. Showalter, "The legal status of autonomous underwater vehicles," Mar. Techn. Soc. J., vol. 38, no. 1, pp. 80-83, 2004.

[19] M. R. Benjamin, J. J. Leonard, J. A. Curcio, and P. M. Newman, "A method for protocol-based collision avoidance between autonomous marine surface craft," J. Field Robot., vol. 23, no. 5, pp. 333-346, 2006.

[20] T. Statheros, G. Howells, and K. McDonald-Maier, "Autonomous ship collision avoidance navigation concepts, technologies and techniques," J. Navigation, vol. 61, no. 1, pp. 129-142, 2008.

[21] C. Wunsch, The Ocean Circulation Inverse Problem. USA: Cambridge University Press, 1996.

[22] A. Shevyrnogova and G. Vysotskaya, "Long-term dynamics of chlorophyll concentration in the ocean surface layer (by space data)," Advances in Space Research, vol. 39, no. 1, pp. 197-202, Jan. 2007.

[23] P. D. Fucile, R. C. Singer, M. Baumgartner, and K. Ball, "A self contained recorder for acoustic observations from AUV's," in Proc. MTS/IEEE Conf. Oceans'06, Boston, MA, USA, Sep. 2006.

[24] P. M. DiGiacomo, L. Washburn, B. Holt, and B. H. Jones, "Coastal pollution hazards in southern California observed by SAR imagery: stormwater plumes, wastewater plumes, and natural hydrocarbon seeps," Marine Pollution Bulletin, vol. 49, pp. 1013-1024, 2004.

[25] J. G. W. Kelley, D. W. Behringer, H. J. Thiebaux, and B. Balasubramaniyana, "Assimilation of SST data into a real-time coastal ocean forecast system for the U.S. East Coast," Weather and Forecasting, vol. 17, pp. 670-690, Aug. 2002. 